\title{
TcruziDB, an Integrated Database, and the WWW Information Server for the Trypanosoma cruzi Genome Project
}

\section{Wim Degrave $/{ }^{+}$, Antonio B de Miranda, Alex Amorim, Adeílton Brandão, Martin Aslett*, Mark Vandeyar**}

\begin{abstract}
Departamento de Bioquímica e Biologia Molecular, Instituto Oswaldo Cruz, Av. Brasil 4365, 21045-900 Rio de Janeiro, RJ, Brasil *European Bioinformatics Institute (EBI), Hinxston, UK **Av. Couronnement 27, 1200

Brucels, Belgium
\end{abstract}

Data analysis, presentation and distribution is of utmost importance to a genome project. A public domain software, $A C e D B$, has been chosen as the common basis for parasite genome databases, and a first release of TcruziDB, the Trypanosoma cruzi genome database, is available by ftp from ftp:// iris.dbbm.fiocruz.br/pub/genomedb/TcruziDB as well as versions of the software for different operating systems (ftp://iris.dbbm.fiocruz.br/pub/unixsoft/). Moreover, data originated from the project are available from the $W W W$ server at http://www.dbbm.fiocruz.br. It contains biological and parasitological data on CL Brener, its karyotype, all available T. cruzi sequences from Genbank, data on the ESTsequencing project and on available libraries, a T. cruzi codon table and a listing of activities and participating groups in the genome project, as well as meeting reports. T. cruzi discussion lists (tcruzil@iris.dbbm.fiocruz.br and tcgenics@iris.dbbm.fiocruz.br) are being maintained for communication and to promote collaboration in the genome project.

Key words: TcruziDB - genome database - Trypanosoma cruzi - clone CL Brener - Parasite Genome Projects anonymous ftp - listserver

Parasite genome projects, some of which started in 1994 after the WHO/TDR sponsored Parasite Genome Network Planning Meeting at Fiocruz (Rio de Janeiro, Brazil, 14-15 April 1994), can be considered as highly successful. Scientists from developing and developed countries have planned and initiated the projects and several "consortiums" for the mapping and sequencing of these mediumsized genomes were established, often based on already ongoing North-South and South-South collaborations. Thus, the genomes of Plasmodium falciparum, Schistosoma mansoni, Trypanosoma cruzi, Leishmania major, Trypanosoma brucei, Brugia malayi, amongst others, are now under study (see http://www.ebi.ac.uk/parasites/parasitegenome.html). The main objectives for these projects are to increase drastically the knowledge

Financial support: UNDP/World Bank/WHO Special Programme for Research and Training in Tropical Diseases; CYTED-Subprogram of Biotechnology (Spain), $\mathrm{CNPq}$, Centro Brasileiro Argentino de Biotecnologia (CBAB/CABBIO).

${ }^{+}$Corresponding author. Fax: +55-21-590.3495. E-mail: wdegrave@gene.dbbm.fiocruz.br

Received 20 August 1997

Accepted 10 September 1997 on the (molecular) biology of these parasites, to identify new genes with key cellular functions, which could be eligible as target for new drugs or new antigens for use in diagnostics or vaccine development, and to promote technology transfer and cooperation between the different participating laboratories.

The $T$. cruzi genome initiative has been working along these lines, and recent reviews on scientific progress have been published (Degrave et al. 1997, Zingales et al. 1997). More than 20 collaborating centers are contributing to the initiative, of which the majority are located in Latin-America, where Chagas disease is endemic. During the first two years of the project, emphasis has been laid on the structuring of the network, acquisition of adequate basic infrastructure for genome research and expertise in new techniques, construction of genomic and cDNA libraries and further characterization of the parasite, including its karyotype. The project has now entered into a large scale sequencing phase, both on ESTs and selected chromosomes, and we can expect the generation of large quantities of new data during the next few years.

However, one of the critical activities in a genome project deals with bioinformatics. It is widely recognized that this field, usually somewhat neglected, will become a major area of study in the 
next decade. Indeed, information, such as nucleotide sequences and maps, are quite useless if it is not analyzed, processed and presented to the greater scientific community.

\section{RESULTS AND DISCUSSION}

TcruziDB, an integrated database for the T. cruzi genome project - At the WHO/NCBI Parasite Genome Computing Workshop, held at Woods Hole Marine Biological Laboratory (Sept. 14-16, 1995), it was decided that the ACeDB software, an object oriented database manager specifically developed for large-scale molecular biology, such as genome projects, would be adopted for the parasite genome projects. The ACeDB software was written initially for the Caenorhabditis elegans project (Durbin \& Thierry-Mieg 1991), but has since then been adapted for a great number of other projects (for an overview, see the ACeDB-faq at http://probe.nalusda.gov/acedbfaq.html), including several plant and parts of the human genome project. ACeDB is an object-oriented database with tools for displaying and analyzing most of the types of objects used in molecular biology and genome projects, such as genes, antigens, genetic maps, sequences, clones, contigs, filter grids, literature data and authors, collaborating colleagues etc. The database can be adapted for the specific needs and information from a certain genome project, and data can be cross referenced where needed according to the "model". User guides can be obtained from http://probe.nalusda.gov:8000/acedocs/. A major drawback of the ACeDB software is that it runs on either UNIX or Linux systems or on Macintosh computers, under $\mathrm{X}$ windows, hampering its widespread use in developing countries. The recent release of Winace, a version running on personal computers with the Win95 or WinNT operating system, should be considered as a major breakthrough.

The first version of the TcruziDB v 1.1 genome database in ACeDB format (version 4.3i) was created and currently contains the following data (Fig. 1):

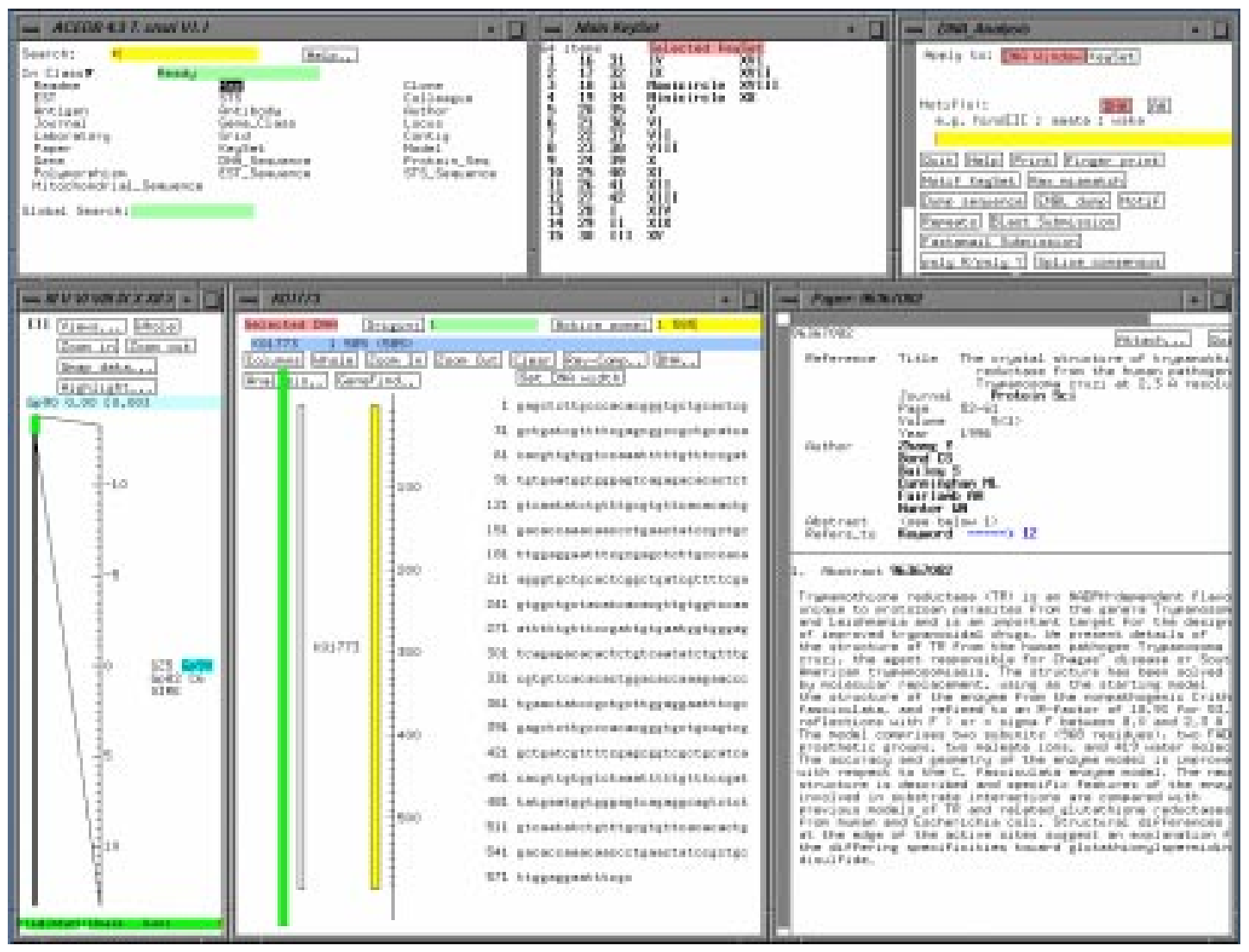

Fig. 1: typical screen of TcruziDB. In the upper left: the main panel with clickable objects. Upper middle: main keyset screen, showing elements of the map object (chromosomal bands in this case). Upper right: the DNA analysis command screen. Lower left: graphical representation of a chromosome. Lower middle: graphical representation of a chromosome fragment and the nucleotide sequence of a mapped gene. Lower right: typical screen of a bibliographic reference. 
- T. cruzi CL Brener chromosomes (numbered I$\mathrm{XX}$ and $1-42$, to reflect the karyotype analyses), as well as minicircle/maxicircle molecules - Mapped loci (50 entries)

- T. cruzi sequences from GB and EMBL (279 entries)

- T. cruzi medline references since 1966 (3573 entries)

- Addresses and data on the collaborators in the $T$. cruzi genome initiative

- T. cruzi protein data from Swissprot.

Ace files for data entry were created through awk and modification of existing perl scripts. The "model" used for this release is similar to the one used for LeishDB, with some modifications. TcruziDB, as well as the databases from the other parasite genome projects, is available via $\mathrm{ftp}$ at $\mathrm{ftp}$ // /iris.dbbm.fiocruz.br/pub/genomedb/TcruziDB. ACeDB software for a variety of operating systems (UNIX, Linux and also Winace) are available at the same site in the directory /pub/unixsoft/.

A second version of TcruziDB v2.1 will be available by the time of this publication and will include updates on the sequences (650 entries), EST sequences (1100 entries or more), medline references, protein data from Swissprot and TREMBL and on collaborators, and will also include new data on filter grids for cosmid and BAC libraries, and fasta alignments. Version 2.1 has modifications in the "model", in order to include pictures and particular data from the T. cruzi project.
The DBBM/IOC biotechnology and genome information server - The WWW server of the Department for Biochemistry and Molecular Biology/Oswaldo Cruz Institute (http:// www.dbbm.fiocruz.br; Fig. 2) offers information and links on genome projects, on some tropical diseases and on general biotechnology, as well as on nucleotide and protein sequence analysis, and provides some general services.

Besides offering links to all major (parasite) genome projects (Fig. 3, Table), the server is the central site for the T. cruzi genome project (Fig. 4), be-

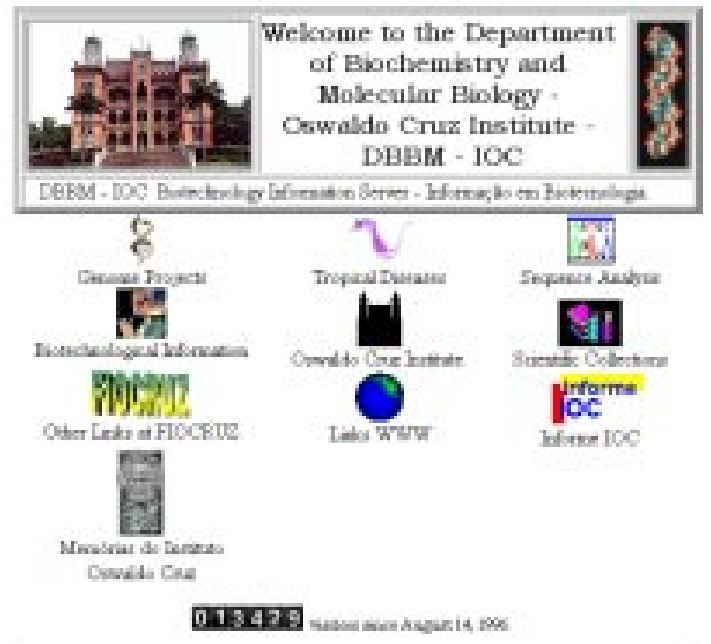

Fig. 2: main page of the DBBM/IOC WWW server.

\section{PARASITE GENOME PROJECTS}

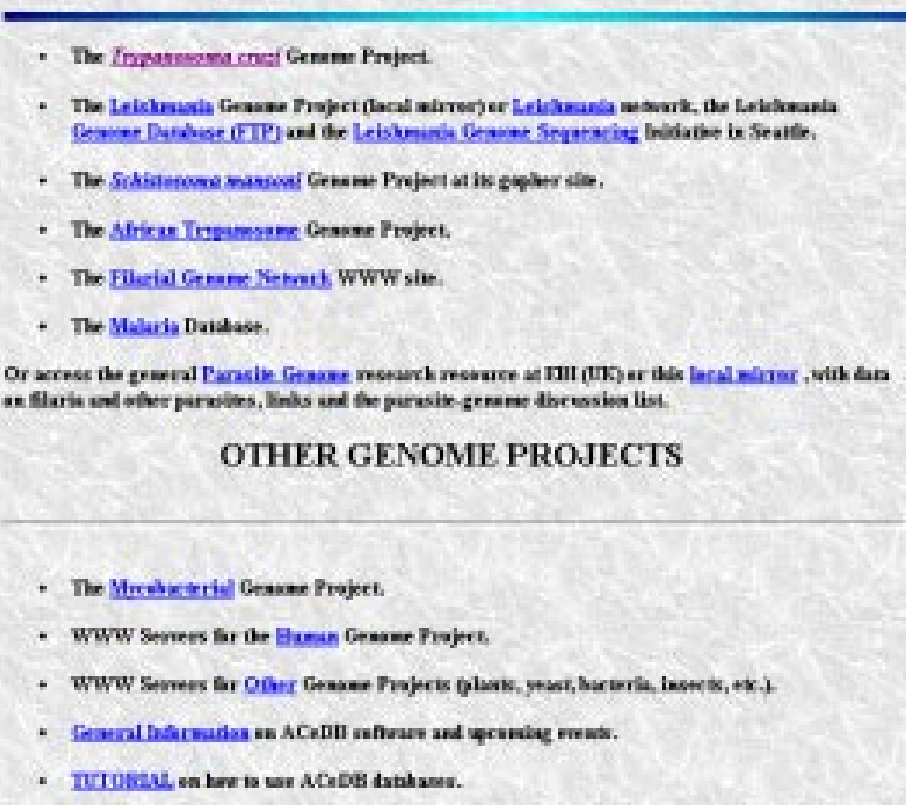

Fig. 3: WWW page with links to genome project web pages. 


\section{TABLE}

Internet access to parasite genome project information servers

\section{Parasite Genome Central Resources}

WWW site

email network

ftp site

Trypanosoma cruzi Genome Project

WWW sites

email network

ftp site

Malaria (Plasmodium) Genome Project

Network WWW sites

email network

Trypanosoma brucei Genome Project

WWW site

ftp site

\section{Leishmania Genome Project}

Genome Project WWW site (UK)

Genome Project WWW site (US)

email network

ftp site

Filarial Genome Project

Genome Project WWW site

email network

ftp site

Schistosoma Genome Project

WWW site

email network

ftp site http://www.ebi.ac.uk/parasites/parasite-genome.html parasite-genome@mailbase.ac.uk ftp://ftp.ebi.ac.uk/pub/databases/parasites

http://www.dbbm.fiocruz.br/genome/tcruzi/tcruzi.html http://www.dbbm.fiocruz.br/tropical/chagas/trypan.html tcruzi-1@iris.dbbm.fiocruz.br (general) tcgenics@iris.dbbm.fiocruz.br (closed list) ftp://iris.dbbm.fiocruz.br/pub/genomedb/TcruziDB

http://www.wehi.edu.au/biology/malaria/who.html http://www.wehi.edu.au/biology/malaria/wellcome.html malaria@wehi.edu.au

http://parsun1.path.cam.ac.uk/newtryp/toppage.htm ftp://ftp.ebi.ac.uk/pub/databases/parasites/brucei/

http://www.ebi.ac.uk/parasites/leish.html http://chimera.biotech.washington.edu/lgnsea.htm LeishL@bdt.org.br ftp://ftp.ebi.ac.uk/pub/databases/parasites/Leish/

http://helios.bto.ed.ac.uk/mbx/fgn/filgen.html filarial-genome@mailbase.ac.uk ftp://ftp.ebi.ac.uk/pub/databases/parasites/Brugia/

http://www.nhm.ac.uk/schisto/ bionet.organisms.schistosoma schistosoma@dl.ac.uk ftp://ftp.ebi.ac.uk/pub/databases/parasites/Schisto/

\section{The Typanosoma cruz/ Genome Initiative}

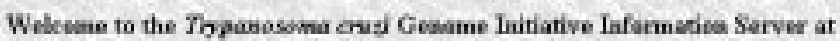
DRBMIOC - FOCR1Z, Rin de Janeirn, Rrasil.

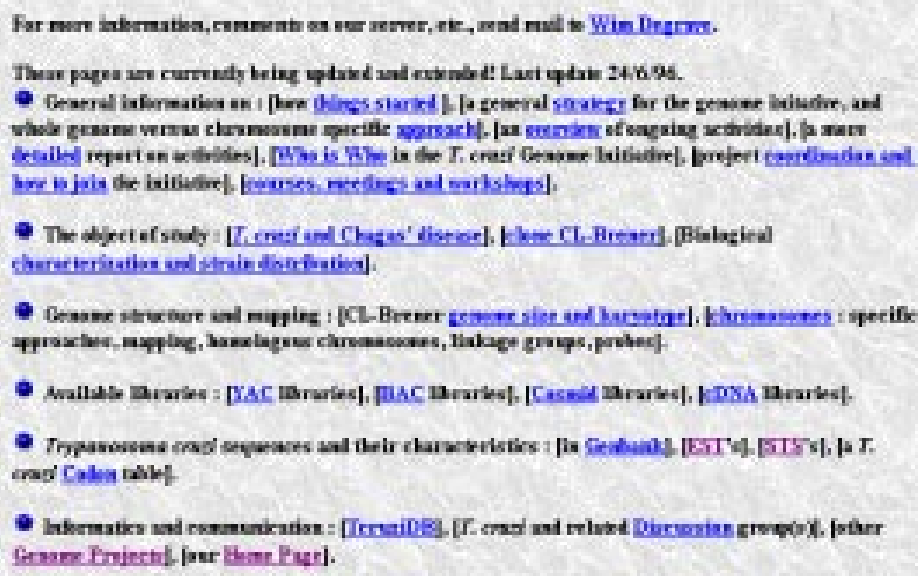

Fig. 4: main page with information on the Trypanosoma cruzi genome project. 
sides mirroring the sites for the project on Leishmania and for the parasite-genome pages. The pages on the T. cruzi genome project present general information, as well as details on available libraries, sequences, and EST data from our laboratory, before deposit to dbEST. Data for the web pages have been gathered through personal contact with the participants of the genome project.

Discussion lists, related to T. cruzi - A T. cruzi discussion list, automated through the majordomo software (subscription by sending a message "subscribe tcruzi-l”to majordomo@ @iris. dbbm.fiocruz.br; messages to tcruzi-1@iris.dbbm. fiocruz.br) is unmoderated and a monthly archive is made. Up to now, about 150 researchers from more than 20 countries are subscribed. The (closed) list tcgenics@iris.dbbm. fiocruz.br has been set up in order to improve the cooperation between the participating groups in the $T$. cruzi genome initiative, and deals specifically with technical communication on the genome project.

\section{REFERENCES}

Degrave W, Levin MJ, da Silveira JF 1997. Parasite genome projects and the Trypanosoma cruzi genome initiative. Mem Inst Oswaldo Cruz (this volume).

Durbin R, Thierry-Mieg J 1991. A C. elegans database. Documentation, code and data available from anonymous ftp servers at lirmm.lirmm.fr, cele.mrclmb.cam.ac.uk and ncbi.nlm.nih.gov.

Zingales B, Rondinelli E, Degrave W, da Silveira JF, Levin M, Le Paslier D, Modabber F, Dobrokhotov B, Swindle J, Kelly JM, Aslund L, Hoheisel JD, Ruiz AM, Cazzulo JJ, Pettersson U, Frasch AC 1997. The Trypanosoma cruzi genome initiative. Parasitol Today 13: 16-22. 
810 TcruziDB, a Database for the T. cruzi Genome Project • W Degrave et al. 